\title{
Die Politik des lebenslangen Lernens in Europa nach dem Boom
}

\author{
Michael Geiss
}

Online publiziert: 30 . August 2017

(C) Der/die Autor(en) 2017. Dieser Artikel ist eine Open-Access-Publikation.

Zusammenfassung Dieser Artikel behandelt die Politik des lebenslangen Lernens in Europa nach 1973. Im Zentrum steht die Frage, inwiefern die 1980er-Jahre als Periode des abnehmenden Interesses am lebenslangen Lernen oder als formative Phase des sogenannten Neoliberalismus interpretiert werden müssen. Auf der Grundlage einer basalen korpuslinguistischen Analyse der offiziellen Überlieferung der Europäischen Gemeinschaften, wie sie die Datenbank EUR-Lex dokumentiert, werden diese Deutungen hinterfragt. Im Anschluss an das theoretische Konzept des ,technology corporatism“ (Bornschier) werden die Befunde stattdessen als Ausdruck eines neuen europäischen Governance-Regimes verstanden, das gemeinsam von supranationalen politischen Akteuren, Großindustrie und Wissenschaft getragen wurde.

Schlüsselwörter Lebenslanges Lernen · Europa · Korpuslinguistik · Technologie · Korporatismus

\section{The European politics of lifelong learning after the boom}

Abstract This article is concerned with the discourse and policy of lifelong learning in Europe after 1973. It deals with the question whether the 1980s have to be seen as a period of diminishing interest in lifelong learning or as a formative phase of so called neoliberalism. Applying a corpus lingustic approach, it analyses the official documents of the European Communities as archived in the database EURLex. The results are interpreted as the expression of a new European governance

Die Analysen zu den in den Organisationen der Europäischen Gemeinschaften verwendeten Begrifflichkeiten erfolgten über die offizielle Datenbank „EUR-Lex“ http://eur-lex.europa.eu/. Die Verteilungen wurden zuletzt am 14. Juni 2017 überprüft.

Dr. M. Geiss $(\bowtie)$

Universität Zürich, Zürich, Schweiz

E-Mail: mgeiss@ife.uzh.ch 
regime called "technology corporatism" (Bornschier), which was jointly supported by supranational political actors, large-scale industry and science and also affected their understanding of lifelong learning.

Keywords Lifelong learning · Europe - Corpus linguistic approach · Technology • Corporatism

\section{Einleitung}

Die erste „Ölpreiskrise“ von 1973 gilt in der zeitgeschichtlichen Forschung mittlerweile als eine wichtige historische Zäsur. Selbst in Deutschland bestimmen nicht länger die Machtübernahme der Nationalsozialisten, Kriegs- und Nachkriegsjahre die Diskussion. Vielmehr sind die Jahrzehnte nach dem „Boom“, den „Trente Glorieuses“, dem „Wirtschaftswunder“ oder „miracolo economico“ ins Zentrum des Interesses gerückt. Damit verbunden ist ein Wechsel der methodischen Zugänge. Im Vergleich zur Politik-, Gesellschafts-, Alltags- oder Kulturgeschichte hat die nationale und transnationale Wirtschaftsgeschichte stark an Bedeutung gewonnen. Die jüngeren Entwicklungen, so die vielfach vertretene Auffassung, lassen sich nicht mehr erfassen, ohne Strukturbruch und Wirtschaftspolitik zu thematisieren. Das Wachstum des Dienstleistungssektors, der Aufstieg der Computertechnologie und die wiederkehrenden Krisen auf dem Arbeitsmarkt bestimmen auch die Gegenwart (Andresen et al. 2011; Ahrens et al. 2013; Reitmayer und Schlemmer 2013; DoeringManteuffel et al. 2016).

Dieser Artikel behandelt die Politik des lebenslangen Lernens in Europa nach 1973. Im Zentrum steht die Frage, wie sich das Bildungsverständnis der supranationalen politischen Akteure in den 1980er-Jahren veränderte. In der Literatur wird dieser Zeitabschnitt bisher entweder als Periode des abnehmenden Interesses am lebenslangen Lernen oder als formative Phase des Neoliberalismus interpretiert (Abschn. 1). Im Anschluss an das theoretische Konzept des ,technology corporatism“ (Bornschier) werden die Befunde im Folgenden als Ausdruck eines neuen europäischen Governance-Regimes verstanden, das gemeinsam von supranationalen politischen Akteuren, Großindustrie und Wissenschaft getragen wurde. Diese Form der Steuerung war eigentlich die europäische Antwort auf den ökonomischen Rückstand im Bereich der Halbleitertechnologie. Entsprechend lag ihr zunächst kein bildungspolitisches Programm zugrunde. Im Rahmen der bisherigen Forschung zum „Technologie-Korporatismus“ wird aber auch wiederholt auf die wachsende Bedeutung des lebenslangen Lernens hingewiesen. Der Beitrag widmet sich diesem Zusammenhang auf der Grundlage einer basalen korpuslinguistischen Auswertung der offiziellen Überlieferung der europäischen Institutionen (Abschn. 2). Die Analysen legen nahe, dass die konzertierten Maßnahmen im Technologiebereich tatsächlich Konsequenzen für das Bildungsverständnis der europäischen politischen Akteure hatten (Abschn. 3). Wie Aus- und Weiterbildung genau verhandelt wurden, wird anschließend durch eine textnahe Auswertung derjenigen Dokumente aus dem Gesamtkorpus rekonstruiert, in denen bildungspolitischen Fragen im Zentrum stehen (Abschn. 4). Für zukünftige Untersuchungen zu klären bleibt, wie sich die for- 
cierte Technologiepolitik zu anderen gesellschaftspolitischen Problemen verhält, die in Europa seit den 1980er ebenfalls durch Maßnahmen im Bereich der Aus- und Weiterbildung gelöst werden sollten (Abschn. 5).

\section{Befunde der Forschung und offene Fragen}

Weiterbildung und lebenslanges Lernen sind noch kaum Gegenstand der jüngeren zeithistorischen Forschung in Deutschland geworden. Das ist insofern erstaunlich, als die soziologischen Zeitdiagnosen einer „Wissens-“ oder „Informationsgesellschaft" hier durchaus Berücksichtigung gefunden haben (Doering-Manteuffel und Raphael 2008). Demgegenüber hat die Erziehungswissenschaft bereits eine Reihe von Arbeiten hervorgebracht, die einen längsschnittlichen Blick auf Weiterbildung und Lebenslanges Lernen erlauben (siehe etwa Weber und Wittpoth 1999; Brine 2002; Davies 2003; Fleige 2007; Schrader 2011). Gut erforscht ist zum einen die Diskursgeschichte des Lebenslangen Lernens (siehe etwa Field 2001; Óhidy 2008). Zum anderen hat sich insbesondere die Weiterbildungsforschung den supra- und transnationalen politischen Akteuren wie der OECD, den Organisationen der Europäischen Gemeinschaften oder der UNESCO gewidmet und dabei je Agenda Setting und Steuerungsinstrumente analysiert (Schemmann und Bruns 2009; Schemmann 2009; Zeuner 2011).

Die Geschichte des Lebenslangen Lernens in Europa ist aus zwei Gründen kompliziert und vielschichtig. Erstens befinden sich die international gebräuchlichen Terminologien in einem beständigen Wechsel. Zum Teil laufen unterschiedliche Begrifflichkeiten parallel - oder derselbe Ausdruck bezeichnet verschiedene Sachverhalte. Zweitens versuchen mehrere internationale Organisationen, dem Diskurs des Lebenslangen Lernens ihren Stempel aufzudrücken. Dabei möchten sie nicht nur konkurrierende Auslegungen durchsetzen, sondern übernehmen mitunter normative Maßstäbe ihrer Mitstreiter. In Europa findet dieser Wettbewerb um die Semantiken nicht zuletzt vor dem Hintergrund unterschiedlicher kultureller Traditionen statt.

Drei trans- bzw. internationale Akteure werden in der Literatur zur Geschichte des Lebenslangen Lernens vor allem berücksichtigt: Die „United Nations Educational, Scientific and Cultural Organization“ (UNESCO), die „Organisation for Economic Co-operation and Development" (OECD) sowie die verschiedenen Organisationen im Rahmen der Europäischen Gemeinschaften. Diese drei Organisationen bzw. Organisationengeflechte strukturierten mit ihrer je unterschiedlichen Ausrichtung für einen gewissen Zeitraum des Zwanzigsten Jahrhunderts die Debatte und konnten wichtige Konzepte einbringen. Die UNESCO steht in dieser Auslegeordnung für einen wissenschaftlich orientierten Humanismus, der in Fragen des Lebenslangen Lernens die Bildung des Subjekts ins Zentrum stellt. Die OECD hingegen erscheint als derjenige Akteur, der klar eine wirtschaftspolitische Agenda verfolgt. Sie gilt im Bildungswesen als Wegbereiter einer ,neoliberalen Regierungsweise“ (Dean 2007, S. 80). Während die UNESCO eine globalistische Perspektive einnimmt, bietet die OECD im Untersuchungszeitraum eine Entwicklungspolitik für die entwickelte Welt an (Tröhler 2013; Bürgi 2017). Bei den Institutionen der europäischen Gemeinschaften liegt der Fall etwas anders. Obwohl sie zur Integration Europas vornehmlich ein 
wirtschaftspolitisches Instrumentarium einsetzten, wies das Projekt normativ darüber hinaus. Im Gegensatz zur UNESCO und zur OECD hatten die europäischen Akteure zudem - wenn auch schwache - Mittel zur politischen Gestaltung. Sie mussten sich also nicht allein auf Beratung, Vernetzung, freiwillige Kooperation und Datenproduktion verlassen, sondern konnten auf eine gewisse politische Legitimation und Durchsetzungskraft ihres Handelns abstellen.

Während diese Konstellation und der unterschiedliche Status der drei Akteure in der Forschung nicht problematisiert werden, sind die Phasen des Diskurses um das Lebenslange Lernen Gegenstand einer anhaltenden Kontroverse. Dabei steht insbesondere die Deutung des Zeitraums zwischen erster Ölpreiskrise und Wiedervereinigung zur Debatte. Alexandra Dehmel identifiziert zwei internationale Hochphasen des Lifelong-Learning-Diskurses. Einen ersten Boom macht sie für die erste Hälfte der 1970er-Jahre aus (Dehmel 2006, S. 50 f.). Andere Autoren setzen den Auftakt bereits einige Jahre früher an (Field 2001; Lee et al. 2008). Markiert wird diese Hochphase in der Forschung durch drei Dokumente. Hierzu zählen der programmatische Text „Permanent Education“ des Europarats (1970), das Strategiepapier „Recurrent Education“ der OECD (CERI 1973), das im Untertitel von der „Lifelong Education“ spricht, und - vor allem - der sogenannte Faure-Report „Learning to be“ der UNESCO (1972), der auch für die europäischen Diskussionen bis heute ein Referenztext ist. Einen zweiten „Peak“ identifizieren Dehmel und andere seit Beginn der 1990er-Jahre, in denen alle drei internationalen Akteure wiederum wichtige Programmpapiere veröffentlichten. Diese Hochphase hatte mit dem „European Year of Lifelong Learning“ (1996) einen klaren Kristallisationspunkt (COM (99) 447 final 1996). Eingeläutet wurde das europäische Jahr des Lebenslangen Lernens 1995 mit einem eigenen White Paper, das zeitdiagnostisch von der ,learning society“ ausging (Dehmel 2006, S. 53). Weil es dem Problem des sozialen Ausschlusses zu wenig Rechnung trug, erfuhr das Programmpapier allerdings ungeahnten Protest (Field 1997). Dennoch ist unbestritten, dass seit Beginn der 1990er-Jahre in unzähligen internationalen Beiträgen auf die Bedeutung von Aus- und Weiterbildung zur Lösung gesellschaftlicher und wirtschaftlicher Problemlagen verwiesen wurde.

Für den Zeitraum zwischen diesen beiden Hochphasen konstatiert Dehmel ein vorwiegend durch die Ölpreiskrise bedingtes ,decreasing interest“ am Lebenslangen Lernen. (Dehmel 2006; siehe auch bereits Schemmann 2002) Vera Centeno (2011) und jüngst Nina Volles (2016) folgen dieser Lesart im Grunde. Bis in die 1960er-Jahre bestimmte der Erwachsenenbildungsdiskurs, wie über das Lernen außerhalb von Familie und obligatorischer Schule diskutiert wurde. Die Bildung des Individuums, Subjektivität und ein starker Humanismus waren die Eckwerte dieses Diskurses, der von den Institutionen der Arbeiterbildung, den Volkshochschulen oder den kirchlichen Trägern gestützt wurde (siehe für die Schweiz auch Geiss 2016). Verkörpert wurde diese Programmatik von der UNESCO, die 1949 in Dänemark erstmals eine „Weltkonferenz über die Erwachsenenbildung“ durchführte (UNESCO 1949, S. 36 ff.).

Auf der zweiten „Weltkonferenz“ 1960 wurde die Erwachsenenbildung zum ,necessary part of the educational provision of every country" erklärt und somit der bildungspolitische Fokus geschärft. Nun standen nicht länger einzelne benachteiligte Gruppierungen im Zentrum. Vielmehr wurde die Qualifikation außerhalb des 
traditionellen Unterrichtswesens nunmehr systematisch angegangen und als Teil des Bildungssystems gedacht. Im Laufe der Vorbereitung zur dritten Konferenz 1972 favorisierte die UNESCO dann einen gemeinsamen Begriff, den der ,éducation permanente“", um das Ziel der eigenen Bestrengungen zu umreißen. An diesem Punkt verließ der Diskurs um das Lebenslange Lernen seine Kopplung an die Tradition der Erwachsenenbildung und bezog sich stattdessen auf alle Lebensbereiche und -phasen (Centeno 2011).

Die gut fünfzehn Jahre zwischen der Euphorie der frühen 1970er und dem langen Jahrzehnt des Lebenslangen Lernens erscheinen bei Centeno als Zeit der semantischen Abkühlung. Explizit widerspricht Centeno Deutungen, diese Verschiebungen allein als Ausdruck veränderter sozioökonomischer Bedingungen zu lesen. Die Ökonomisierung und Individualisierung der Perspektive sei eher als Folge der Verschränkung unterschiedlicher Diskurse zu interpretieren (ebd., S. 140, Fn. 5).

Deutlich wird die These eines „decreasing interests“ in einem Artikel von Moosung Lee, Tryggvi Thayer und Na'im Madyun kritisiert (Lee et al. 2008). Sie begreifen die anderthalb Jahrzehnte vor dem Fall des Eisernen Vorhangs geradezu als formative Phase des Neoliberalismus. Diese habe das selbstgesteuerte Lernen und die Individualisierung von Verantwortung für den eigenen Bildungsprozess das vormals humanistische, an der klassischen Erwachsenenbildung orientierte Verständnis des Lebenslangen Lernens abgelöst.

Wie kommt es aber zu diesen unterschiedlichen Einschätzungen? Keinem der genannten Autorinnen und Autoren ließe sich vorwerfen, dass sie von einem zu kleinen Quellenkorpus ausgehen. Zwar finden sich mitunter anachronistische Argumentationsmuster, wenn von der Kenntnis späterer Ereignisse die Vorgeschichte rekonstruiert wird. Das erklärt aber nicht die verschiedenen Lesarten. Vielmehr hängen diese erstens davon ab, welche Rolle einzelnen Dokumenten zugesprochen wird. Ein Einbruch des Interesses am Lebenslangen Lernen lässt sich dann gut belegen, wenn die Darstellung um den Faure-Report der UNESCO von (1972) und das White Paper der Europäischen Kommission ,Teaching and Learning: Towards the Learning Society" von 1995 (COM 95, 590 final 1995) sowie die verschiedenen Programmschriften des Folgejahrs gruppiert wird. Zweitens spielt es für die Periodisierung der Geschichte des Lebenslangen Lernens eine gewichtige Rolle, welche Bedeutung der verwendeten Terminologie zugeschrieben wird. Wenn die verschiedenen Begrifflichkeiten als Träger fest eingeschriebener Bedeutungen interpretiert werden, liegt die Annahme einer diskontinuierlichen Entwicklung nahe. Vor allem der verschiedentlich konstatierte, in den 1990er-Jahren vollzogene Wechsel von ,education“ zu „learning“ würde dann einen Paradigmenwechsel anzeigen, der mit der Bedeutungszunahme der OECD im Bildungsbereich korrespondiert. Drittens spielt es für die Frage nach der Periodisierung eine gewichtige Rolle, ob ein gemeinsamer Diskurs mit unterschiedlichen Akteuren rekonstruiert werden soll, ob sich die Analyse auf eine der transnationalen Organisationen konzentriert. Lee et al. (2008) haben beide Ansätze miteinander kombiniert, indem sie Prozesse des institutionellen Lernens ins Zentrum stellen. 


\section{Theoretische Verortung und methodische Ausrichtung}

Im Folgenden werde ich einen anderen Weg gehen, indem ich versuche, den spezifischen Kontexten der europäischen Entwicklungen in den 1980er stärker Rechnung zu tragen. Hierbei orientiere ich mich an dem theoretischen Konzept des ,technology corporatism“. Mit diesem Begriff fassen der Soziologe Volker Bornschier und sein Team Verschiebungen im europäischen Governanceregime, die sie für die 1980er-Jahre nachweisen konnten. Anders als ältere Korporatismuskonzepte, die analytisch häufig unscharf (vgl. Geiss 2017) - verschiedene Formen der Mitwirkung demokratisch nicht-legitimierter Körperschaften am politischen Prozess bezeichnen, wird mit dem Begriff eine bestimmte Konstellation im Europäisierungsprozess bezeichnet, die als technologiepolitische Antwort auf den Vorsprung Japans und der USA im Bereich der Neuen Technologien Konsequenzen auch für andere Bereiche des supranationalen politischen Gefüges hatte. Wissenschaft bzw. Technologie war in dieser Form der Korporatismus sowohl als (schwacher) Akteur als auch als (starker) Kontext präsent. Der ,technology corporatism“ ist nicht als eine im strengen Sinne tripartite politische Ordnungsfigur zu verstehen. Die Bedeutung der Informationstechnologien im wirtschaftlichen Wettbewerb mit den USA und Japan machten aber Verschiebungen in der Machtarchitektur der europäischen Gemeinschaften möglich, die sonst kaum denkbar gewesen wären. Der neuen Konkurrenzsituation in der technologischen Entwicklung, bei der die Staaten Europas gegenüber Asien und den USA ins Hintertreffen zu geraten drohten, wurde nicht nur mit einem verstärkten Engagement im Bereich der Forschung und Entwicklung beantwortet. Die Erfindung des Mikrochips stellte auch neue Anforderungen an die Qualifizierung der Arbeitskräfte (Parker 2000, S. 94 f.; Bornschier 1996, S. 241).

Den wirtschaftspolitischen Antworten auf den Strukturwandel wäre entsprechend auch für die Historiographie des lebenslangen Lernens mehr Beachtung zu schenken. Inwiefern der ,technology corporatism“ auch bildungspolitische Konsequenzen hatte, soll hier durch eine basale korpuslinguistische Untersuchung beantwortet werden. Der Fokus liegt damit auf der supranationalen Ebene. Die Untersuchung bezieht mit den Europäischen Gemeinschaften eine Reihe korporativer Akteure ein, die deutlich weniger Handlungsressourcen haben als die nationale Politik, aber mehr Strukturierungsmacht haben als etwa die OECD. (Schrader 2011, S. 99 ff.) Ich werde mich auf die öffentlich zugänglichen Dokumente der europäischen Institutionen konzentrieren und den Schwerpunkt auf die Phase zwischen den beiden „Peaks“ des Diskurses zum lebenslangen Lernen legen. Mich interessiert dabei, inwiefern diese als Periode eines ,decreasing interest“ (Dehmel) oder als „,formative period for the neo-liberal lifelong learning discourse“ (Lee et al.) zu interpretieren sind. Als Quellenbestand dienen mir die in der Datenbank EUR-Lex abgelegten Protokolle, Strategiepapiere, Memoranden und Beschlüsse verschiedener europäischer Akteure. Die Grundgesamtgesamtheit an Dokumenten wurde über eine Anfrage mit dem Wortstamm ,euro*“ konstruiert, da derzeit über das Formular der Datenbank keine Angaben über den Korpusumfang abrufbar sind. Es ist davon auszugehen, dass nahezu alle als Volltext durchsuchbaren Dokumente diesen Wortstamm enthalten. Der Gesamtbestand für den Zeitraum zwischen 1968 und 2000 beläuft sich somit zum Analysezeitpunkt auf 294.059 Einzeldokumente, wobei erst 1972 jährlich mehr 
als 1000 Dokumente abgelegt sind. Ab 1985 sind es dann durchgängig über 10.000 Dokumente.

Die Datenbank EUR-Lex dokumentiert im Kern den Prozess europäischer Rechtsgenese. Ein Großteil der verzeichneten Dokumente sind damit Verordnungen und Richtlinien sowie Vorarbeiten zum europäischen Recht, also parlamentarische Anfragen, Stellungnahmen verschiedener Akteure und Positionierungen der Europäischen Kommission. Letztere zeichnet auch für die meisten der im Gesamtkorpus vorhanden Dokumente verantwortlich. Daneben sind das europäische Parlament und der Rat der europäischen Gemeinschaften stark vertreten. Für bildungspolitische Belange spielt im Rahmen der forcierten Anstrengungen im Technologiebereich in den 1980er-Jahren überdies der Europäische Wirtschafts- und Sozialausschuss eine gewisse Rolle.

Die Suche über die Datenbank wurde nicht vorab nach Akteuren, Dokumentarten oder Art der Publikation eingeschränkt. Gesucht wurde in der Datenbank jeweils nach Wortstämmen, Begriffen oder Begriffskombinationen. Die Ausschläge stehen für die Zahl der Dokumente, in denen der Suchterm jeweils gefunden wurde. Um die Bedeutung der einzelnen Dokumente innerhalb des Gesamtbestandes zu ermitteln, werden sie in Relation zu ihrem jeweiligen Jahrgang gesetzt. So lösen beispielsweise die Funde vor 1972 aufgrund des kleinen Quellenkorpus unverhältnismäßig starke Ausschläge aus, was bei der Interpretation berücksichtigt werden muss.

Um größere Entwicklungen überhaupt nachzeichnen zu können, müssen relativ abstrakte Begrifflichkeiten ausgewählt und diese auf Wortstämme verkürzt werden, die mit einer hohen Wahrscheinlichkeit den gesuchten Sachverhalt auch anzeigen. Deshalb wurde etwa nicht nach „further education“, „lifelong education“ oder ,lifelong learning" gesucht, sondern allgemeiner nach bildungspolitischen Konjunkturen gefragt und etwa auch der Wortstamm ,school*“ einbezogen. Damit die spezifischen Zusammenhänge, in die diese eingebettet sind, nachvollzogen werden können, wurden in einem zweiten Schritt in einer textnahen Rekonstruktion auch diejenigen Dokumente ausgewertet, aus denen auf den Kontext der Begriffsverwendung geschlossen werden kann.

\section{Berufliche Aus- und Weiterbildung in Europa nach der Ölpreiskrise}

Fragt man zunächst einmal unspezifisch nach der Nennung pädagogischer bzw. bildungspolitischer Termini wie ,educat*“, „learn*“, ,school*“ oder ,training*“ zeigt sich ein erstaunliches Resultat. Von 1974 und 1990 beträgt der Anteil der Dokumente, die mindestens einen dieser Begriffe enthalten, durchgängig zwischen gut sechs und gut acht Prozent. Seit der Korpusumfang eine gewisse Größe erreicht hat, gibt es bis in die 1990er-Jahre keine großen Ausschläge bzw. Einbrüche, sondern eine relativ kontinuierliche Diskussion bildungspolitischer Fragen. In den 1990erJahren verdoppelt sich dann der Anteil der Dokumente, die auf Schule, Aus- und Weiterbildung verweisen (Abb. 1).

Die zwei Jahrzehnte vor den 1990er-Jahren können als ein intensiviertes Engagement im Bildungsbereich interpretiert werden: Die Bildungsminister der zu diesem Zeitpunkt noch kleinen Europäische Gemeinschaft hatten 1971 eine stärkere 
Abb. 1 Results containing educat* OR training* OR learn* OR school* in title and text, from 1968 to 2000. Quelle: EUR-Lex 2017

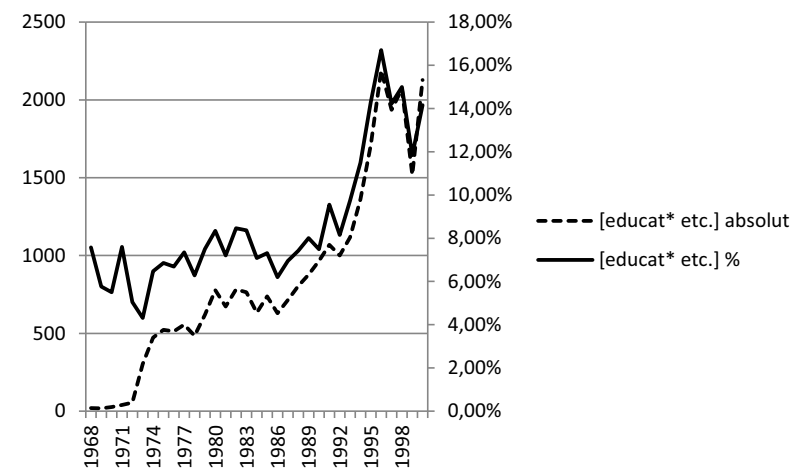

Kooperation im Bildungswesen vereinbart. Ziel war, die ökonomische Integration Europas durch ein kulturelles Modell zu komplementieren. Als ein Meilenstein galt der 1976 verabschiedete Aktionsplan, der zwar nur ein verhältnismäßig kleines Budget zur Umsetzung hatte, dafür aber systematisch auf einer ganzen Reihe von Feldern gemeinsame Anstrengungen in der Bildungspolitik vorsah. Eine koordinierte Bildungspolitik schien so dringlich zu sein, dass auf europäischer Ebene zahlreiche Tätigkeiten initiiert wurden. Durch ständigen Austausch von Erfahrungen und Informationen wollte man die Bildungssysteme auf ein gemeinsames Ziel hin ausrichten (European Commission 2006, S. 63 ff.).

Auf einer abstrakten Ebene hebt sich das Engagement der europäischen Organisationen in den 1990er-Jahren deutlich von dem der vorigen Jahrzehnte ab. Dies ist als Folge des Vertrags von Maastricht und den Bestrebungen im Bereich des Lebenslangen Lernens zu interpretieren. Über den gesamten Untersuchungszeitraum verteilt ist die Zahl derjenigen Dokumente am höchsten, die mindestens den Begriff ,training*“ aufweisen, gefolgt von dem Wortstamm ,educat*“. Der Wortstamm ,school*“ und „learn*“ findet sich hingegen deutlich seltener. Im Zeitverlauf lässt sich zeigen, dass der Lernbegriff erst in den 1990er-Jahren überhaupt etwas an Bedeutung gewinnt. Im Vergleich zu ,training“ bleibt dieser aber selbst dann noch marginal (Abb. 2 und 3). Daraus wird deutlich, dass Fragen der wirtschaftlich relevanten beruflichen Aus- und Weiterbildung von den europäischen Akteuren durchgängig das größte Gewicht beigemessen wird.

Abb. 2 Total results containing educat*, training*, learn*, school* in title and text, from 1968 to 2000. Quelle: EUR-Lex

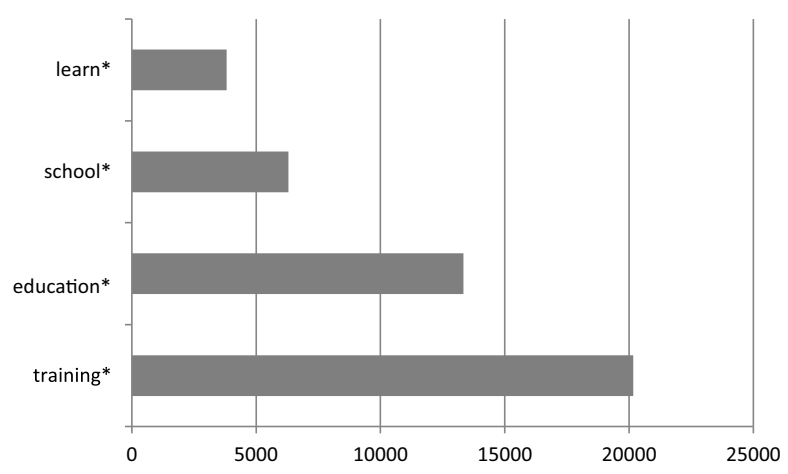


Abb. 3 Results containing educat*, training*, learn*, school* in title and text, from 1968 to 2000. Quelle: EUR-Lex

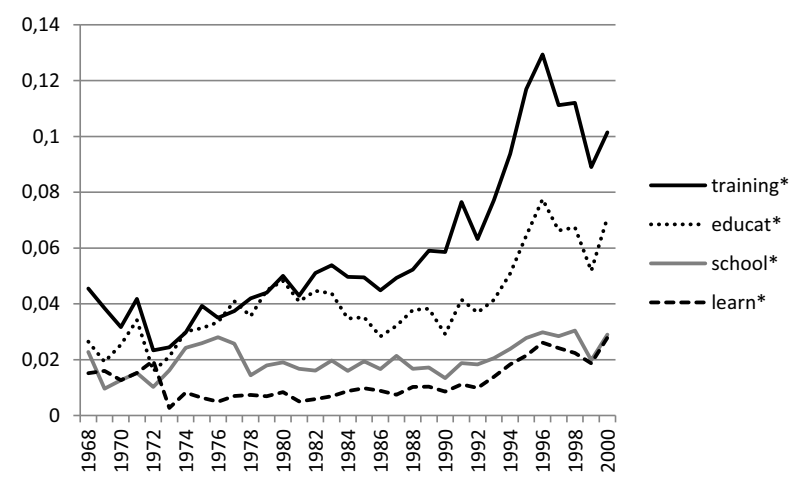

Die Einbrüche zu Beginn des Untersuchungszeitraums sind aufgrund des geringen Umfangs des Gesamtkorpus vorsichtig zu interpretieren. Während für „training“ eine langsame ansteigende quantitative Bedeutung auszumachen ist, lässt sich an „education“ das in der Literatur wiederholt konstatierte abnehmende Interesse in den 1980er-Jahren ablesen. Historiographisch lässt sich dieser Sachverhalt aber auch so interpretieren, dass der starke Anstieg in den 1990er-Jahren die vorgängigen Entwicklungen unterschätzt. Blickt man vom Jahr 1996 ausgehend retrospektiv auf die 1970er und 1980er-Jahre und misst den zentralen Dokumenten der europäischen Organisationen, der OECD und der UNESCO eine große Bedeutung zu, können die anschließenden Entwicklungen nur als ein „decreasing interest“ interpretiert werden. Die längsschnittliche Sicht auf die Begriffsverwendungen erlaubt hingegen eine andere Gewichtung.

Was also geschah genau in den 1980er-Jahren? Hierfür lohnt es sich, eine weitere Begrifflichkeit einzuführen, die in der Literatur nur als eine wichtige Einflussgröße neben anderen genannt wird: die der Technologie. Über den Untersuchungszeitraum lässt sich eine starke Bedeutungszunahme technologiepolitischer Ansätze ausmachen. Europäische Technologiepolitik war zwar in den 1980er-Jahren zunächst nicht zwingend auch Bildungspolitik. Die Zahl der Dokumente, die sich mit Fragen der Technologie beschäftigen und dabei nicht auf Aus- und Weiterbildung verweisen, ist im gesamten Untersuchungszeitraum höher als diejenige mit Bildungsbezug, die aber ebenfalls an Gewicht gewinnt. Zudem zeigt sich die überproportionale Bedeutungszunahme bildungspolitischer Fragen für die 1990er-Jahre auch hier, wobei der Abstand zu den rein technologiepolitisch ausgerichteten Papieren wieder zunimmt (Abb. 4).

Das, was von Lee et al. als formative Phase des Neoliberalismus bezeichnet wird, erscheint eher als Vorbereitung einer forcierten Wirtschaftspolitik im Sinne des ,technology corporatism“, bei der auch Aus- und Weiterbildung eine Rolle spielen. Dies entspricht einem Befund der zeithistorischen Forschung, dass sich in der Periode, in der gesellschaftspolitisch auf die Krise zunehmend mit Deregulierung und Staatsabbau reagiert wurde, zugleich in einer bisher Weise staatliches Kapital mobilisiert wurde, um die Entwicklung von Neuen Technologien voranzutreiben. Europa sah sich durch die Innovationen Nordamerikas und Japans zunehmend bedroht (OECD 1981; Straumann 2001). 
Abb. 4 Results containing technolog* AND [educat* OR training* OR learn* OR school*]; Results containing technolog NOT [educat* OR training* OR learn* OR school*] in title and text, from 1968 to 2000. Quelle: EUR-Lex
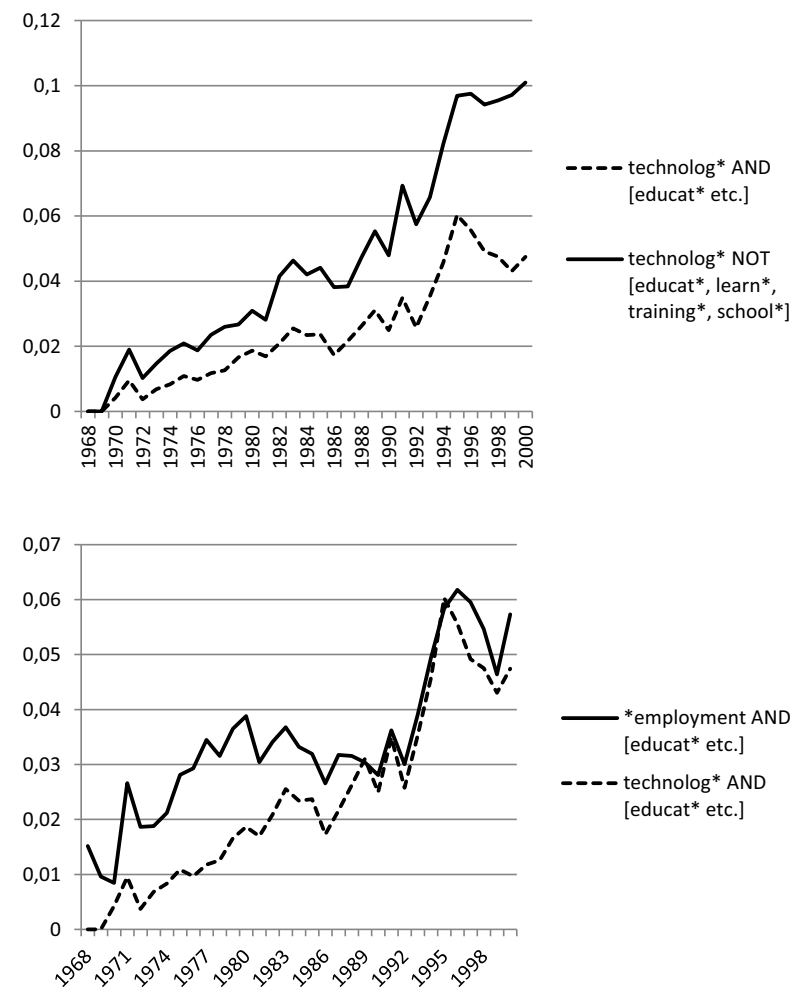

Abb. 5 Results containing technolog* AND [educat* OR training* OR learn* OR school*]; results containing employment AND [educat* OR training* OR learn* OR school*] in title and text, from 1968 to 2000. Quelle: EUR-Lex

Die Lösung der Folgen der Ölpreiskrisen mochte Technologiepolitik sein, das Problem hieß aber Arbeitslosigkeit. Vergleicht man die Zahl der Dokumente mit Bildungbezug auf das Verhältnis technologie- und beschäftigungspolitischer Argumente hin, fällt auf, dass die Arbeitsmarktthematik zunächst die bildungspolitische Agenda stärker bestimmt, sich die Kurven aber im Laufe der 1980er-Jahre angleichen. Mit der Stärkung der Wettbewerbsfähigkeit der europäischen Mitgliedsländer sollten die Folgen der Rezession auf dem Arbeitsmarkt eingefangen werden. Mit dem Fokus auf Technologie wurde kurzfristigen konjunkturpolitischen Maßnahmen eine strukturpolitische Alternative entgegengesetzt (Abb. 5).

\section{Mikrochip, Arbeitsmarkt und, ,lifelong training“}

Um das Verhältnis von Technologiepolitik, lebenslangem Lernen und Weiterbildung genauer zu rekonstruieren, reicht die basale Korpuslinguistik nicht aus. Notwendig ist vielmehr eine textnahe Analyse derjenigen Dokumente, die sich explizit Fragen der Aus- und Weiterbildung widmen: 1974 forderte der Rat der Europäischen Gemeinschaften die Europäische Kommission auf, sich verstärkt mit den Herausforderungen durch die elektronische Datenverarbeitung auseinanderzusetzen. Dieser wurde große Bedeutung für alle gesellschaftlichen Bereiche attestiert. Für das Beste- 
hen im globalen Wettbewerb schien das Engagement in diesem Bereich intensiviert werden zu müssen. Nur durch eine verstärkte Zusammenarbeit meinte man, die Firmen in Europa in die Lage versetzen zu können, mit den anderen Akteuren zu konkurrieren. Gemeinsame Standards, die Mobilisierung qualifizierter Arbeitskräfte und kooperativ entwickelte Anwendungen galten als Ziel der gemeinsamen Anstrengungen (Official Journal of the European Communities vom 20. Juli 1974).

Das Problem der Neuen Technologien wurde zunächst nicht unter bildungspolitischen Gesichtspunkten verhandelt. Die Stellungnahme des einflussreichen, mit verschiedenen Stakeholdern besetzten Europäischen Wirtschafts- und Sozialausschuss zu den vorgeschlagenen Maßnahmen in diesem Bereich enthält 1975 keine Vorschläge zur Verbesserung der Aus- und Weiterbildung. Ein Papier aus demselben Jahr zur Bildungspolitik weist umgekehrt explizit das Ansinnen zurück, die Bildungspolitik allein unter dem Gesichtspunkt der Bereitstellung wissenschaftlichen und technischen Personals zu diskutieren (Official Journal of the European Communities vom 7. November 1975 sowie vom 17. November 1975).

Bereits wenig später drängte der Europäische Wirtschafts- und Sozialausschuss darauf, den Fragen der Aus- und Weiterbildung ein größeres Gewicht in der Technologiepolitik beizumessen. Er monierte das vollständige Fehlen von Qualifizierungsansätzen in den Vorschlägen der Kommission zur elektronischen Informationsindustrie (Official Journal of the European Communities vom 16. Februar 1977; COM 77, 283 1977) und forderte, dass den ,opportunities, limitations and possible dangers of these new techniques“ viel stärker Rechnung zu tragen sei. Der Ausschuss ging von massiven Veränderungen der Wirtschaftsstruktur durch die wachsende Bedeutung der Informatik aus, weshalb der Bildung der Nutzer ein besonderes Augenmerk gelten müsse (Official Journal of the European Communities vom 23. Januar 1978, S. 3).

Der Europäische Wirtschafts- und Sozialausschuss kam zu dem Schluss, dass die durch die Neuen Technologien geschaffenen Stellen ausschließlich im Bereich des hochqualifizierten Personals angesiedelt sein würden. Für alle anderen Bereiche müsse davon ausgegangen werden, dass der Strukturwandel Jobs vernichte. Um das Problem angehen zu können, sollten umfassende Studien in Auftrag gegeben werden, die besonders den Möglichkeiten zur Umschulung Rechnung trügen (Official Journal of the European Communities vom 23. Januar 1978, S. 4 f.). Bereits zu diesem Zeitpunkt wurden also in die technologiepolitische Debatte verschiedene sozialpolitische Aspekte der Aus- und Weiterbildung eingeschrieben. Neben die Qualifikation des technischen und wissenschaftlichen Personals gesellten sich Umschulungsmaßnahmen für diejenigen, die vom Strukturwandel unmittelbar betroffen sein würden.

In der Folge eines Treffens der Staats- und Regierungschefs in Bonn im Juli 1978 benannte der Rat der Europäischen Gemeinschaften explizit die notwendige Weiterbildung von Ingenieuren und Technikern als zentrale Voraussetzung für die Anwendung der Halbleitertechnik in unterschiedlichen industriellen Bereichen und forderte die Kommission auf, entsprechend tätig zu werden (Official Journal of the European Communities vom 13. September 1979). Die Kommission legte anschließend ein Strategiepapier vor, das der Aus- und Weiterbildung in der Technologiepolitik wirklich Rechnung trug: „European society faced with the challenge 
of new information technologies: a Community response" (1979). Die Kommission übernahm hier die Zeitdiagnose der „Informationsgesellschaft“. Diese wurde nicht als eine bloße Zukunftsvision, sondern bereits wurde als Merkmal der Gegenwart verstanden. Man ging davon aus, dass kein Bereich des wirtschaftlichen und gesellschaftlichen Lebens von den Folgen der Erfindung des Mikrochips unberührt bleiben würde. Die Bilder, die der Report zeichnete, waren dramatische Zuspitzungen. Tempo und Reichweite, Effizienzgewinn und Kostenersparnis, Vernetzung und Produktivität - kein Superlativ wurde ausgelassen, um die Dringlichkeit des Themas zu veranschaulichen (COM 79, 650 final 1979, S. 1 f.).

Mit ihren drastischen Zeitdiagnosen war die Kommission nicht allein, wie eine zeitgenössische Bibliographie zur sozialen und ökonomischen Bedeutung der Neuen Technologien zeigt (Grayson 1984). Die Kommission hielt eine forcierte Politik im Bereich der Informationstechnologien für unabdingbar, um die Wettbewerbsfähigkeit der Mitgliedsländer zu erhalten. Beschäftigungspolitisch deutete sie vorsichtig an, dass neben den wegfallenden, eher repetitiven Jobs auch neue Tätigkeitsbereiche entstehen könnten. Ob die neuen Möglichkeiten zum Wohle der Menschheit eingesetzt würden oder nur der Zentralisierung von wirtschaftlicher oder politischer Macht dienten, entscheide sich jetzt (COM 79, 650 final 1979, S. 3).

Als Konkurrenten im Wettbewerb um die Vormachtstellung bei den Informationstechnologien galten den europäischen Institutionen die USA und Japan. Auf Seite der Forschung sah man zwar durchaus, dass die europäischen Forschungsinstitute mithalten könnten. Doch in der kommerziellen und industriellen Nutzung der der intellektuellen Ressourcen schien man weit hinter den anderen beiden Staaten zurückzuliegen. In der Computerindustrie und Chipproduktion wurden die größten Defizite ausgemacht. Bei Software und Telekommunikation sah die Situation etwas besser aus. Den Vorsprung Japans und der USA sah die Kommission aber mitnichten zunächst in einer anderen Wirtschafts- oder Forschungsstruktur begründet. Vielmehr galten hier die nationalen Subventionen als Hauptgrund für ihre führende Position auf dem Weltmarkt. Auch diejenigen Staaten Europas, die über relativ starke Unternehmen im Bereich der Informationstechnologie verfügten, hätten ihre Position nur aufgrund von umfangreichen Staatsinvestitionen. Die unterschiedlichen nationalen Standards erschwerten zusätzlich, dass sich ein gemeinsamer europäischer Markt für die Neuen Technologien herausbilde. Dasselbe gelte, hier macht das Kommissionspapier einen bemerkenswerten metaphorischen Sprung, für den ,,common market of ideas or the mobility of talent" (COM 79, 650 final 1979, S. $4 \mathrm{f}$.).

Den erwarteten sozialen Folgen der Durchsetzung der Informationstechnologien auf verschiedenen gesellschaftlichen und wirtschaftlichen Feldern sollte durch die Bereitstellung der entsprechenden Fähigkeiten (,skills“) begegnet werden. Mit einem Ausbildungs- und Qualifikationsprogramm für Schulen und Betriebe sowie der Verbreitung des notwendigen Wissens wollte man dem diagnostizierten Strukturwandel begegnen. Der Fluchtpunkt dieser sehr unspezifischen Programmatik der Kommission war eine Politik des Lebenslangen Lernens, mit einer Erhöhung des Qualifikationsniveaus bei den technischen Berufen und mehr Eigeninitiative für den individuellen Bildungsverlauf: ,,and this will call for efforts in the way of education at all ages and of lifelong training on a scale far beyond what is being done today“ (COM 79, 650 final 1979, S. B20). 
Das 1970 eingerichtete „Standing Committee on Employment“, in dem Vertreter der Arbeitgeber und Arbeitnehmer ebenso Einsitz hatten wie Kommissions- und Ratsmitglieder, reagierte auf dieses Papier mit einem eigenen Memorandum. Der bloßen pragmatischen Umschulung erteilte dieses eine Absage und plädierte stattdessen für eine umfassende Neuorientierung der beruflichen Aus- und Weiterbildung (Commission of the European Communities 1980, S. 3), Die Kommission gab entsprechend eine Studie in Auftrag, die sich dem Zusammenhang von Mikroelektronik und beruflicher Bildung widmen sollte. Christopher F. Hayes und Fiona EdwardsStuart, die für den Bericht verantwortlich zeichnete, bemühten sich, nicht mehr den drohenden Wegfall von Arbeitsplätzen ins Zentrum zu stellen, sondern stattdessen auf die Potenziale der Neuen Informationstechnologien hinzuweisen. Insbesondere erteilten sie allen Prognosen eine Absage, die auf der Grundlage dürftiger Daten eine strukturbedingte Massenarbeitslosigkeit von geringqualifizierten Personen diagnostizierten. Grundsätzlich gingen Hayes und Edwards-Stuart davon aus, dass weder das Verhältnis von formaler Ausbildung und Arbeitsmarkt im Bereich der Neuen Technologien noch die genauen Bedürfnissen der Mikrochipindustrie noch die zukünftigen Entwicklungen der Automatisierung immer weiterer Arbeitsbereiche gegenwärtig überhaupt zu bestimmen seien (Hayes und Edwards-Stuart 1980, S. 2 f.).

Hayes und Edwards-Stuart sahen in den großen strukturellen Umwälzungen der Arbeitswelt dennoch eine gewaltige bildungspolitische Herausforderung. So sehr sie bei Prognosen der Dequalifizierung und Rationalisierung zur Vorsicht gemahnten, so sicher waren sie sich, dass sich die Qualifikationserwartungen an den Einzelnen stark verändern würden. Insbesondere sagten sie voraus, dass sich die Unterscheidung von Fähigkeiten, die bei der Arbeit zum Einsatz kämen, und Fähigkeiten, die für das außerberufliche Leben relevant seien, mehr und mehr verwässern würde. Die Prozesse der Arbeitswelt würden dann aber quer zu der tradierten beruflichen Ordnung laufen, wovon auch die Organisation der Unternehmen tangiert sei. Die Schulen könnten sich nicht mehr auf die Vermittlung von Kenntnissen beschränken, sondern müssten den abstrakten Kompetenzen wie Planung, Kommunikation oder Team Work mehr Gewicht zumessen (Hayes und Edwards-Stuart 1980, S. iii, S. $23 \mathrm{f}$.).

Technologiepolitik galt von nun an als das zentrale Instrument, um den Auswirkungen der Rezession auf dem Arbeitsmarkt zu begegnen. Die sozialen Folgen der Neuen Technologien wurden nicht als ein Schicksal begriffen, dem man sich zu fügen hatte, sondern als eine Herausforderung, der durch eine konzertierte Aktion zu begegnen sei. Man ging davon aus, dass die Veränderungen in der Wirtschaftsstruktur nachhaltig und tiefgreifend seien und sich auf die Art der Produktion, der Arbeitsorganisation, der Arbeitsbedingungen, das Verhältnis von Arbeit und Freizeit und die Ausgestaltung der Produkte und Dienstleistungen auswirken würden (COM 82, 296 final 1982, S. 1 f.).

Diese Flexibilisierung der Arbeitswelt schien auch die angestammten Formen der schulischen und beruflichen Ausbildung in Frage zu stellen. Die Weiterbildung des qualifizierten Personals vor allem im technischen und wissenschaftlichen Bereich sowie die Umschulung derjenigen Arbeitskräfte, die fortan nicht mehr hinreichend qualifiziert sein würden, galten als probate Mittel, der unsicheren Zukunft 
zu begegnen. Die Folgen für die Ausgestaltung der Bildungssysteme wurden als „inescapable“ betrachtet (COM 82, 296 final 1982, S. 12).

Im Sommer 1985 legte die Kommission dann einen Aktionsplan mit dem Titel „Community programme in education and training for technology“ (COMETT) vor, das einen Katalog von Maßnahmen für die Zeit von 1986 bis 1992 definierte. In zwei Phasen sollte eine Zusammenarbeit von Hochschulen und Unternehmen angestoßen und auf diese Weise dafür gesorgt werden, dass die notwendigen Qualifikationen zur Verfügung standen und auch direkt genutzt würden. In den ersten drei Jahren wurden unter COMETT 1300 Projekte durchgeführt, 125 Partnerschaften von Industrie und Hochschulen aufgebaut, über 4000 Studierende in den Unternehmen anderer Staaten untergebracht, 232 Stipendien vergeben und 329 Projekte zur Weiterqualifikation unterstützt (Official Journal of the European Communities vom 13. September 1985; COM 93, 151 final 1993; SEC 91, 1016 final 1991).

COMETT war das erste rein bildungspolitische Aktionsprogramm, das durch die neuen technologischen Entwicklungen angestoßen worden war. Es ergänzte das umfassende ,European Strategic Programme of Research and Development in Information Technology“ (ESPRIT), mit denen die Europäische Kommission den technologischen Rückstand im Bereich der Hard- und Software Entwicklung im Bereich der elektronischen Datenverarbeitung aufholen wollte. Bei ESPRIT kam es zu einer bisher nicht gekannten Zusammenarbeit von politischen Akteuren, Wissenschaft und Industrie, die letztlich auch eine Zentralisierung von Kompetenzen über die Technologiepolitik hinaus möglich machte. Dieser ,technologische Korporatismus“ lief auf forcierte Anstrengungen im Bereich der beruflichen und betrieblichen Weiterbildung hinaus, so dass Maßnahmen im Bildungsbereich zukünftig als zentraler Faktor zur Etablierung eines gemeinsamen europäischen Marktes im Zeichen des Mikrochips erschienen (Bornschier 2000; Parker 2000; Geiss 2017).

Das zweite (1987-1991) und dritte (1990-1994) „Framework Programme for Research and Technological Development" sah ebenfalls jeweils Maßnahmen im Bereich der Aus- und Weiterbildung vor. Letzteres enthielt ein Unterprojekt zu „Human capital and mobility“, das explizit als Ergänzung zu COMETT gedacht war. Der Fokus der bildungspolitischen Anstrengungen der europäischen Institutionen wurde aber schnell über die engere Technologie- und Wissenschaftspolitik hinaus erweitert. Mit dem ,European Region Action Scheme for the Mobility of University Students“ seit 1987 (ERASMUS), dem ,Action Programme for the vocational training of young people and their preparation for adult and working life" (PETRA) oder dem „European Network of Vocational Training Projects for Women“ (IRIS) seit 1988 wurden andere Anspruchsgruppen als nur das hochqualifizierte wissenschaftliche und technische Personal in den Blick genommen. YOUTH FOR EUROPE ebenfalls seit 1988 oder LINGUA und TEMPUS seit 1990 stellten Mobilität und Fremdsprachen ins Zentrum, nun auch unter veränderten politischen Bedingungen. Die Technologiepolitik blieb aber ein wichtiger Treiber der europäischen Aktionsprogramme. Das „Action programm to promote innovation in the field of vocational training resulting from change in the European Community" (EUROTECNET) seit 1990 und das ,Action Programme for the development of continuing vocational training in the European Community“ (FORCE) seit 1991 zielten direkt auf die Verbindung von Technologie und veränderter Aus- und Weiterbildung. Bereits 1988 
war eine Fortsetzung des COMETT-Programms verabschiedet worden, das 1990 startete und sich als eine Vertiefung und Erweiterung der früheren Ansätze verstand (COM 93,151 final 1993; COM 93, 409 final 1993).

\section{Resümee und Ausblick}

In der Literatur zur Politik des lebenslangen Lernens werden die 1980er-Jahre entweder als Periode des abnehmenden Interesses an bildungspolitischen Fragen oder als formative Phase des Neoliberalismus verstanden. Beide Lesarten sind nicht falsch, bedürfen aber einer Rekontextualisierung. So verschob sich in dieser Zeit der Fokus der supranationalen Akteure in Europa hin zu einer technologiepolitischen Agenda, mit der die Mitgliedsstaaten wieder wettbewerbsfähig gemacht werden sollten. Die verschiedenen europäischen Akteure sahen sich durch die technologischen Entwicklungen im Bereich der elektronischen Datenverarbeitung unter massiven Handlungsdruck gesetzt. Gegenüber Japan und den USA drohten die nationalen Volkswirtschaften ins Hintertreffen zu geraten. Eine Harmonisierung von Standards und Märkten, größere Mobilität der Arbeitskräfte, forcierte Anstrengungen in Forschung und Entwicklung sowie eine Reform der Aus- und Weiterbildung galten als probate Mittel, um diesem Problem Herr zu werden. Im Laufe der 1980er-Jahre wurde diese Diagnose nur noch selten in Frage gestellt.

Nicht mehr kurzfristige konjunkturpolitische Maßnahmen standen im Zentrum, sondern eine nachhaltige Strukturpolitik, die den Unternehmen dienen und die grassierende Arbeitslosigkeit abbauen sollte. Neben Forschung und Entwicklung galten Aus- sowie verschiedene Formen der Weiterbildung als entscheidende Instrumente, um den neuen Herausforderungen zu begegnen. Formativ für die 1990er-Jahre war das vorgehende Jahrzehnt insofern, als hier unter technologie- und wirtschaftspolitischen Gesichtspunkten eine Politik des lebenslangen Lernens formuliert wurde, die alle gesellschaftlichen Gruppierungen, alle pädagogischen Institutionen und alle Formen der Aus- und Weiterbildung betraf. Nicht individuelle Vervollkommnung oder Gemeinwohl waren die Leitmaximen dieses Diskurses, sondern eine Stärkung der Wettbewerbsfähigkeit Europas im Zeichen des Strukturwandels. Der Mikrochip, der die Grenzen von Arbeit und Freizeit, privat und öffentlich, Mensch und Technologie zunehmend auflösen sollte, verflüssigte auch die tradierten Unterschiede im Bildungswesen. Schulische Bildung, die klassische Berufslehre oder tradierte Formen der Weiterqualifikation schienen allzu starre Gebilde zu sein, um dem rapiden Wandel gerecht zu werden, mit dem sich nicht nur die europäischen Staaten konfrontiert sahen.

Die Anstrengungen der europäischen Gemeinschaften und ihrer Mitgliedsländer im Aus- und Weiterbildungsbereich erschöpften sich nicht in den hier skizzierten Entwicklungen. Die Verschiebung von einer auf kurzfristige Maßnahmen abzielenden Beschäftigungspolitik hin zu einer langfristigen Strukturpolitik aber, wie sie im Bereich der Jugendarbeitslosigkeit ebenfalls nachzuweisen ist, gewann unter dem Gesichtspunkt der Neuen Technologien an Dringlichkeit. Der Wechsel von einer reaktiven und feldspezifischen hin zu einer koordinierten und übergreifenden Arbeitsmarkt- und Bildungspolitik hatte im Mikrochip ein ikonisches Moment, das die 
unterschiedlichen Stränge zusammenhielt. Mit den Neuen Technologien erschien eine konzertierte europäische Bildungspolitik auf allen Ebenen und für alle Bevölkerungsgruppen einerseits unabdingbar. Andererseits galt ständige Weiterqualifikation nicht nur als Antwort, sondern selbst als Teil eines gewaltigen strukturellen und alternativlosen Wandels (Neave 1988, S. 109 ff.).

$\mathrm{Zu}$ untersuchen bleibt, wie sich die beschriebenen Entwicklungen zu anderen Problemzusammenhängen der beruflichen Aus- und Weiterbildung verhalten: Langzeitund Jugendarbeitslosigkeit, die Öffnung qualifizierter Beschäftigung für Frauen, die Integration vom Menschen mit Behinderung in den Arbeitsmarkt oder Fragen der Alphabetisierung waren im Untersuchungszeitraum ebenfalls Gegenstand bildungspolitischen Handelns. Inwiefern sie durch die technologiepolitische Wende einen Schub erhielten oder durch diese bedroht wurden, wäre zu untersuchen. Auch bleibt zu rekonstruieren, inwiefern bzw. auf welche Weise die Tätigkeiten der europäischen Akteure ihren Niederschlag in den Mitgliedsstaaten fanden. Zuletzt bietet es sich an, dem Übergang von einer forcierten Wirtschafts- und Technologiepolitik hin zu einer Politik des Lebenslangen Lernens in den 1990er-Jahre stärker Beachtung zu schenken. Dass dabei der wirtschaftliche Strukturwandel als zeitdiagnostische Rahmung und als erlebte Realität eine Rolle spielte, scheint mehr als wahrscheinlich zu sein.

Danksagung Ich danke Urs Grob für seine Unterstützung und Johanna Egli sowie der Redaktion und den anonymen Gutachtern für ihre wertvollen Hinweise zu einer früheren Fassung des Artikels.

Open Access Dieser Artikel wird unter der Creative Commons Namensnennung 4.0 International Lizenz (http://creativecommons.org/licenses/by/4.0/deed.de) veröffentlicht, welche die Nutzung, Vervielfältigung, Bearbeitung, Verbreitung und Wiedergabe in jeglichem Medium und Format erlaubt, sofern Sie den/die ursprünglichen Autor(en) und die Quelle ordnungsgemäß nennen, einen Link zur Creative Commons Lizenz beifügen und angeben, ob Änderungen vorgenommen wurden.

\section{Literatur}

Ahrens, R., Gehlen, B., \& Reckendrees, A. (Hrsg.). (2013). Die „Deutschland AG“. Historische Annäherungen an den bundesdeutschen Kapitalismus. Essen: Klartext.

Andresen, K., Bitzegeio, U., \& Mittag, J. (Hrsg.). (2011). Nach dem Strukturbruch? Kontinuität und Wandel von Arbeitsbeziehungen und Arbeitswelt(en) seit den 1970er-Jahren. Bonn: Dietz.

Bornschier, V. (1996). Western society in transition. New Brunswick: Transaction.

Bornschier, V. (2000). Western Europe's Move toward Political Union. In V. Bornschier (Hrsg.), Statebuilding in Europe: the revitalization of Western European integration (S. 3-37). Cambridge: Cambridge University Press.

Brine, J. (2002). Further education participation, European expansion and European erasure. British Educational Research Journal, 28(1), 21-36.

Bürgi, R. (2017). Die OECD und die Bildungsplanung der freien Welt: Denkstile und Netzwerke einer internationalen Bildungsexpertise. Opladen: Budrich.

Centeno, V. (2011). Lifelong learning: a policy concept with a long past but a short history. International Journal of Lifelong Education, 30(2), 133-150.

CERI (1973). Centre for educational research and innovation: recurrent education: a strategy for lifelong learnin. Paris: OCDE.

COM (1977). Decisions to be taken. The common policy in the field of science and technology, Bd. 1283 final.

COM (1979). European society faced with the challenge of new information technologies: a community response. 650 final.

COM (1982). Vocational training and new information technologies: new community initiatives during the period 1983-1987. 296 final. 
COM (1993). EC education \& training programmes 1986-1992. Results and achievements: an overview. Report from the Commission to the Council, the European Parliament and the Economic and Social Committee. 151 final.

COM (1993). Report from the commission to the council, the European Parliament and the Economic and social committee: COMETT. Programme, Report of 1992 Activities. 409 final.

COM (1995). White paper on education and training - teaching and learning - towards the learning society. 590 final.

COM (99) 447 final (1996). Implementation, results and overall assessment of the European Year of Lifelong Learning (1996). Report from the Commission to the Council, the European Parliament, the Economic and Social Committee and the Committee of the Regions submitted in accordance with Article 8 of European Parliament and Council Decision No 2493/95/EC, 15 September 1999.

Commission of the European Communities (1980). Employment and the new micro-electronic technology. Information memo. Brüssel: Commission of the European Communities.

Davies, P. (2003). Widening participation and the European union: direct action, indirect policy? European Journal of Education, 38, 99-116.

Dean, M. (2007). Die „Regierung von Gesellschaften“. Über ein Konzept und seine historischen Voraussetzungen. In S. Krasmann \& M. Volkmer (Hrsg.), Michel Foucaults „, Geschichte der Gouvernementalität" in den Sozialwissenschaften. Internationale Beiträge (S. 74-104). Bielefeld: transcript.

Dehmel, A. (2006). Making a European area of lifelong learning a reality? Some critical reflections on the European union's lifelong learning policies. Comparative Education, 42(1), 49-62.

Doering-Manteuffel, A., \& Raphael, L. (2008). Nach dem Boom. Perspektiven auf die Zeitgeschichte seit 1970. Göttingen: Vandenhoeck \& Ruprecht.

Doering-Manteuffel, A., Raphael, L., \& Schlemmer, T. (2016). Vorgeschichte der Gegenwart: Dimensionen des Strukturbruchs nach dem Boom. Göttingen: Vandenhoeck \& Ruprecht.

EUR-Lex (2017). Access to European Law. European Union, Luxembourg, 1998-2017. eur-lex.europa.eu. Zugegriffen: 14.06.2017.

European Commission (2006). The history of European cooperation in education and training: Europe in the making, an example. Luxembourg: Office for Official Publications of European Communities.

Field, J. (1997). The learning society and the European Union: a critical assessment of supranational education policy formation. Journal of Studies in International Education, 1(2), 73-92.

Field, J. (2001). Lifelong education. International Journal of Lifelong Education, 20(1-2), 3-15.

Fleige, M. (2007). Veränderungen des Geschlechterverhältnisses in der Weiterbildung in Deutschland - Weiterbildungsbeteiligung und Angebotsentwicklung 1980-2003. Hessische Blätter für Volksbildung, 3, 221-231.

Geiss, M. (2016). Sanfter Etatismus. Weiterbildungspolitik in der Schweiz. In L. Criblez, Ch Rothen \& Th Ruoss (Hrsg.), Staatlichkeit in der Schweiz. Regieren und Verwalten vor der neoliberalen Wende (S. 219-246). Zürich: Chronos.

Geiss, M. (2017). Technologiepolitik und die Ordnung der beruflichen Weiterbildung. In K. Manz, F. Imlig \& L. Lehmann (Hrsg.), Folgeprobleme. Annäherungen an eine Theorie der Schulreform. Wiesbaden: Springer VS.

Grayson, L. (Bearb.) (1984). The social and economic impact of new technology 1978-84: a select bibliography. Letchworth: Technical Communications.

Hayes, C., \& Edwards-Stuart, F. (1980). Micro-electronics and vocational training in the European Community. Study no 80/35. Brüssel: Europäische Kommission. Studie im Auftrag der EG-Kommission.

Lee, M., Tryggvi, T., \& Na'im, M. (2008). The evolution of the European union's lifelong learning policies: an institutional learning perspective. Comparative Education, 44(4), 445-463.

Neave, G. (1988). Policy and response: changing perceptions and priorities in the vocational training policy of the EEC commision. In G. J. Lauglo \& K. Lillis (Hrsg.), Vocationalizing education: an international perspective (S. 99-114). Oxford: Pergamon.

OECD (1981). Organisation for economic co-operation and development. Science and technology policy for the 1980 s. Paris: OECD.

Óhidy, A. (2008). Lifelong learning. Interpretations of an education policy in Europe. Wiesbaden: Springer VS.

Parker, S. (2000). Esprit and technology corporatism. In V. Bornschier (Hrsg.), State-building in Europe: the revitalization of Western European integration (S. 93-121). Cambridge: Cambridge University Press.

Reitmayer, M., \& Schlemmer, T. (2013). Die Anfänge der Gegenwart Umbrüche in Westeuropa nach dem Boom. München: Oldenbourg. 
Schemmann, M. (2002). Lifelong learning as a global formula. In K. Harney, A. Heikkinen, S. Rahn \& M. Schemmann (Hrsg.), Lifelong learning: one focus, different systems (S. 23-31). Frankfurt am Main: Peter Lang.

Schemmann, M. (2009). Erklärungsansätze für die Entstehung und Verbreitung internationaler Weiterbildungspolitik zwischen World Polity und transnationalem Raum. Hessische Blätter für Volksbildung, $2,116-122$

Schemmann, M., \& Bruns, H. (2009). Der Einfluss der Europäischen Union auf den Weiterbildungssektor - zwischen Steuerung und Governance. Zeitschrift Außerschulische Bildung, 4, 360-367.

Schrader, J. (2011). Struktur und Wandel der Weiterbildung. Bielefeld: Bertelsmann.

SEC (1991). Comett I. Final report of the commission (1986-1990). 1016 final.

Straumann, T. (2001). Rezession, Technologiepolitik und Risikokapital. Das Scheitern der Innovationsrisikogarantie, 1985. Schweizerische Gesellschaft für Wirtschafts- und Sozialgeschichte, 17, 403-419.

Tröhler, D. (2013). Standardisierung nationaler Bildungspolitiken. Die Erschaffung internationaler Experten, Planer und Statistiken in der Frühphase der OECD. International Journal for the Historiography of Education, 3(1), 60-77.

UNESCO (1949). United nations educational, scientific and cultural organization. Summary Report of the International Conference on Adult Education. Paris: Unesco.

UNESCO (1972). United nations educational, scientific and cultural organization. Learning to be: the world of education today and tomorrow. Paris: Unesco.

Volles, N. (2016). Lifelong learning in the EU: changing conceptualisations, actors, and policies. Studies in Higher Education, 41(2), 343-363.

Weber, K., \& Wittpoth, J. (1999). Discourse, structure and practice of continuing education: a comparison between Switzerland and Germany. International Review of Education, 45(5/6), 547-560.

Zeuner, C. (2011). Internationale Perspektiven der Erwachsenenbildung. In R. Tippelt \& A. von Hippel (Hrsg.), Handbuch Erwachsenenbildung/Weiterbildung (S. 583-598). Wiesbaden: Springer VS.

\section{Weiterführende Literatur}

Council of Europe (1970). Permanent education: a compendium of studies commissioned by the council for cultural cooperation. Strasbourg: Council of Europe.

Official Journal of the European Communities, diverse Jahrgänge. 